\title{
Evaluation of PLAGA/n-HA Composite Scaffold Bioactivity in vitro
}

\section{Qing Lv ${ }^{1,2}$, Xiaohua Yu ${ }^{1,4}$, Meng Deng ${ }^{1,2,4}$, Lakshmi S. Nair' ${ }^{1-5}$ and Cato T Laurencin ${ }^{1-6^{*}}$}

${ }^{1}$ Institute for Regenerative Engineering, University of Connecticut Health Center, School of Medicine, Farmington, CT 06030, USA

${ }^{2}$ The Raymond and Beverly Sackler Center for Biomedical, Biological, Physical and Engineering Sciences, University of Connecticut Health Center, School of Medicine,

Farmington, CT 06030, USA

${ }^{3}$ Department of Biomedical Engineering, University of Connecticut, School of Engineering, Storrs, CT 06268, USA

${ }^{4}$ Department of Orthopaedic Surgery, University of Connecticut Health Center, School of Medicine, Farmington, CT 06030, USA

${ }^{5}$ Department of Chemical and Biomolecular Engineering, University of Connecticut, School of Engineering, Storrs, CT 06268, USA

${ }^{6}$ Department of Materials Science and Engineering, University of Connecticut, School of Engineering, Storrs, CT06268, USA

\begin{abstract}
Polymeric sintered microsphere scaffolds have shown their tremendous potential in bone tissue engineering applications due to their highly porous and interconnected three dimensional structure and excellent mechanical properties. While these scaffolds are able to support basic cellular activity after seeding cells on them, the bioactivity of these scaffolds in terms of enhancing the biological performance of stem cells during bone regeneration is still under satisfactory. We hypothesized that incorporation of bioactive addictive such as hydroxyapatite into these scaffolds could improve their bioactivity without sacrificing the bulk properties of the scaffolds. We have successfully incorporated nano-hydroxyapatite ( $\mathrm{n}-\mathrm{HA}$ ) into poly (lactic acid-glycolic acid) (PLAGA) microsphere based scaffolds in our previous studies. Herein, we aimed to evaluate the bioactivity of PLAGA/n-HA composite scaffolds, with a focus on studying the mineralization of the scaffolds in vitro. The capability of inducing apatite formation in vitro was largely enhanced in the composite scaffolds compared to plain PLAGA scaffolds. More importantly, PLAGA/n-HA composite scaffolds have been shown to improve rabbit mesenchymal stem cells (RMSCs) proliferation, differentiation, and mineralization as compared to control PLAGA scaffolds. Taken together, introduction of n-HA appears to be an efficient approach to improve the bioactivity of PLAGA scaffolds for bone tissue engineering.
\end{abstract}

Keywords: Composite scaffold; Poly (Lactic Acid Glycolic Acid); Nano hydroxyapatite; Bone tissue engineering; Bioactivity

\section{Introduction}

Scaffolds as a key component in bone tissue engineering should meet a series of criteria, such as appropriate porous structure, bioactivity, appropriate degradation rate with non-toxic degradation products, and cytocompatibility [1-3]. Towards the design and development of ideal scaffold for optimal regenerative performance, a variety of scaffold fabrication techniques such as solvent casting/salt leaching, phase separation, and freeze-drying have been employed to generate scaffolds with distinct properties [4-6]. Although all these techniques have demonstrated their potential for bone tissue engineering application, a microsphere-based approach for tissue engineering initially developed by Laurencin et al. appears to be extraordinarily attractive [7]. Scaffold generated by this method could provide highly inter-connected $3 \mathrm{D}$ structure as well as sufficient mechanical properties close to functional trabecular bone $[8,9]$. The primary choice of materials for this technique is so far poly (lactic acid-glycolic acid) (PLAGA), a biodegradable polymer which has been most widely investigated and FDA approved for various biomedical applications [10,11]. While microsphere-based scaffolds made of PLAGA have shown to be able to support basic cellular activity in/on the scaffolds such as cell attachment and proliferation $[12,13]$ their bioactivity in terms of guiding cell differentiation towards osteogenic lineage is still considered poor.In order to improve the biological performance of these scaffolds, incorporation of bioactive components which provide cells with inductive cues into the scaffolds is highly desirable in the context of bone tissue engineering.

Hydroxyapatite has been well established as excellent osteo conductive materials due to its similarity to the inorganic components of natural bone [14]. It has been widely used as an addictive to form composite materials with both synthetic and natural polymers $[15,16]$. Recently, nano hydroxyapatite (n-HA) attracted significant attention as the exceptionally large surface area of these particles provides extraordinary performance as the second phase added into the scaffolds $[17,18]$. We hypothesized that incorporation of n-HA into the sintered PLAGA microsphere-based scaffolds could greatly improve the bioactivity of the scaffold. Previously, PLAGA/n-HA composite scaffolds have been successfully fabricated in our laboratory $[19,20]$. In this study, we focused on the evaluation of the bioactivity of these composite scaffolds compared to PLAGA scaffolds. Both acellular mineralization and cell based mineralization assays were employed to fully characterize the biological performance of the scaffolds in vitro.

\section{Materials and Methods}

\section{Materials}

PLAGA(85:15 lactic acid to glycolic acid ratio, $\mathrm{MW}=120 \mathrm{kDa})$ was obtained from Lakeshore Biomaterials (Wilmington, $\mathrm{OH}$ ). n-HA particles were purchased from Berkeley Advanced Biomaterials (San Leandro, CA) with average diameter around $100 \mathrm{~nm}$. All other reagents were purchased from Sigma-Aldrich (St. Louis, MO) unless otherwise stated.

\section{Preparation and characterization of PLAGA/n-HA composite microspheres}

PLAGA/n-HA composite microspheres were prepared via a

*Corresponding author: Cato T, Laurencin, University of Connecticut Health Center, School of Medicine, Farmington, CT 06030; E-mail: Laurencin@uchc.edu

Received March 16, 2014; Accepted October 27, 2014; Published November 03, 2014

Citation: Lv Q, Yu X, Deng M, Nair LS, Laurencin CT (2014) Evaluation of PLAGA n-HA Composite Scaffold Bioactivity in vitro. Bioceram Dev Appl 4: 080. doi: 10.4172/2090-5025.1000080

Copyright: () 2014 Lv Q, et al. This is an open-access article distributed under the terms of the Creative Commons Attribution License, which permits unrestricted use, distribution, and reproduction in any medium, provided the original author and source are credited. 
modified emulsion and evaporation method described previously [21]. Briefly, PLAGA was dissolved in dichloromethane to make a $20 \%$ (w/v) solution. n-HA particles were dispersed into this solution at a PLAGA/n-HA ratio of 4:1 (w/w) and vortexed overnight to form a homogeneous mixture. The composite microspheres were then formed by gradually pouring the PLAGA/n-HA mixture into a $1 \%(\mathrm{w} / \mathrm{v})$ poly (vinyl alcohol) (PVA) solution stirring at $250 \mathrm{rpm}$ (Figure 1). The emulsion system was kept stirring for $24 \mathrm{~h}$ to completely evaporate all the organic solvent. The resultant microspheres were collected after filtering through ashless filter paper, washed with DDI water, and air dried. The dried microspheres were passed through standard sieves and stored in desiccator for future use.PLAGA microspheres without n-HA was also prepared accordingly to serve as control group.

\section{Composite scaffold fabrication and degradation characterization}

Cylindrical composite scaffolds $(4.0 \mathrm{~mm} \times 2.5 \mathrm{~mm})$ were fabricated by a microsphere sintering method described previously. PLAGA/nHA microspheres were packed into a stainless steel mold and sintered at $90^{\circ} \mathrm{C}$ for $3 \mathrm{~h}$. PLAGA sintered microsphere control group was prepared by sinteringPLAGA microspheres at $85^{\circ} \mathrm{C}$ for $3 \mathrm{~h}$.

\section{In vitro mineralization on PLAGA/n-HA composite scaffolds}

Simulated body fluid (SBF) was prepared as reported previously [22]. The reagents were added to distilled and de-ionized (DDI) water in the following order and concentration: $142 \mathrm{mM} \mathrm{NaCl}, 5 \mathrm{mM} \mathrm{KCl}, 1.5$ $\mathrm{mM} \mathrm{MgCl}, 0.5 \mathrm{mM} \mathrm{MgSO}_{4}, 150 \mathrm{mM} \mathrm{NaHCO}, 20 \mathrm{mM}$ Tris, $2.5 \mathrm{mM}$ $\mathrm{CaCl}_{2}$, and $1.0 \mathrm{mM} \mathrm{Na}_{2} \mathrm{HPO}_{4}$. The $\mathrm{pH}$ of $\mathrm{SBF}$ was adjusted to 7.40 using $\mathrm{HCl} / \mathrm{NaOH}$ at $37^{\circ} \mathrm{C}$. Both the composite and plain PLAGA scaffolds were incubated in SBF for 28 days. Control scaffolds for both groups were incubated in DDI water. The solutions were changed every other day. After incubation for 28 days, samples $(n=3)$ were removed from solutions, washed with DDI water, and air-dried for further analysis. The surface morphology and calcium deposition were examined by scanning electron microscopy (SEM).

Calcium deposition was characterized by Alizarin red staining. In brief, the scaffolds were removed from solution, washed with DDI water 3 times, and transferred into new well plates. Scaffolds were stained with $10 \%$ Alizarin red (Sigma, St Louis, MO) solution for 10 minutes. The scaffolds were then washed with DDI water 5 or more times until no color could be washed off. Pictures of stained scaffolds were taken by stereo microscope (Discovery V12, Zeiss). Next, $1.0 \mathrm{~mL}$ $10 \%$ cetylpyridinium chloride (CPC) (Sigma-Aldrich, St. Louis, MO) solution was added to each scaffold to dissolve the color. The optical density of the solution was read at $550 \mathrm{~nm}$ with a TECAN SpectroFluo Plus reader (Boston, MA). The values of absorbance of scaffolds in SBF were reported after subtracting the reading of control groups in DDI water.

\section{RMSCs culture}

Rabbit mesenchymal stem cells (RMSCs)were harvested from New Zealand white rabbits with the protocol approved by the Animal Care and Use Committee, University of Virginia. Briefly, Young male New Zealand White (NZW) rabbits (average $1.67 \mathrm{~kg}$ ) were euthanized using an overdose of sodium pentobarbital. Rabbit bone marrow was then isolated from the tibial and femoral bones and re-suspended in $10 \mathrm{~mL}$ Dulbecco's Modified Eagle's Medium (DMEM). The bone marrow suspension was layered on $10 \mathrm{~mL}$ Ficoll-Paque Plus (Amersham
Biosciences) reagent in a $50 \mathrm{ml}$ conical tube and centrifuged at 1500 $\mathrm{rcf}$ for $35 \mathrm{~min}$. The mononuclear layer (low density) was collected and washed twice with PBS. The mononuclear cells were plated in basal DMEM media with $10 \%$ FBS and $1 \%$ penicillin/streptomycin in flasks. Non-adherent cells were removed when medium was changed after 3 days. The culture medium was then changed 3 times a week. The RMSCs were used till passage 7.

\section{Cell culture on PLAGA/n-HA composite scaffolds}

Scaffolds were soaked in $70 \%$ ethanol for 15 minutes, washed with sterile water twice for 15 minutes each time, and further sterilized by UV irradiation for 30 minutes on each side. RMSCs were seeded onto scaffolds at a density of $5 \times 10^{4}$ cells per scaffold in 96 well plates in basal media. After 24 hours, the scaffolds were transferred into DMEM supplemented with $0.1 \mu \mathrm{M}$ dexamethasone, $50 \mu \mathrm{g} / \mathrm{mL}$ ascorbic acid and $10 \mathrm{mM} \beta$-glycerophosphate. The media were changed every 3 days, and the cultures were maintained for 21 days. At day 7, 14, and 21, scaffolds were taken out for further characterization.

Cell proliferation was measured via application of Cell Titer $96^{\text {ma }}$ Aqueous One Solution Cell Proliferation Assay (MTS Assay) (Promega, Madison, USA). At predetermined time points, the scaffolds were taken out, washed with PBS, and transferred into a 24 well plate. The assay was performed by adding $200 \mu \mathrm{lMTS}$ reagent into each well containing one scaffold and $1 \mathrm{ml}$ cell culture medium. After 2-hour incubation at $37^{\circ} \mathrm{C}, 250 \mu \mathrm{L} 10 \%$ sodium dodecyl sulfate (SDS) solution was added to stop the reaction. The mixture was diluted 5 times and then read at 490 $\mathrm{nm}$ by a Tecan SpectroFluo Plus reader (Boston, MA).

Alkaline phosphatase (ALP) activity was measured as an early marker of osteoblastic phenotype. At each time point, scaffolds were taken out, washed with PBS, and transferred into a new well plate. Cells were lysed by adding $1.0 \mathrm{~mL} 1 \%$ Triton X-100 solution and then subjected to 3 freeze-thaw cycles. The resultant cell lysates were stored at $-80^{\circ} \mathrm{C}$ for further assay. At the end of culture period, the cell lysate samples were thawed together and assayed. A $100 \mu \mathrm{L}$ sample was mixed with $400 \mu \mathrm{L}$ substrate solution (a mixture of $p$-Nitrophenylphosphate, diethanolamine buffer and DI water) and incubated at $37^{\circ} \mathrm{C}$ for $30 \mathrm{~min}$. $100 \mu \mathrm{L} 0.4 \mathrm{M} \mathrm{NaOH}$ was added to stop the reaction and the resultant solution is read at $405 \mathrm{~nm}$ using the TECAN reader. The ALP activity was reported as absorbance. The cell mediated mineralization on both types of scaffolds was analyzed using Alizarin red staining and quantified as described in the previous sections.



Figure 1: Schematic of the PLAGA/n-HA microsphere fabrication process using an emulsion and solvent evaporation method 


\section{Results and Discussion}

\section{Degradation behavior of PLAGA/n-HA composite scaffolds}

Mineralization of PLAGA/n-HA composite scaffolds in vitro: PLAGA and PLAGA/nHA sintered microsphere scaffolds were incubated in SBF to test their mineralization capability in vitro. After incubation in SBF for 28 days, samples were taken out and examined under SEM. The SEM micrographs showed the difference in the surface morphology between PLAGA/n-HA and PLAGA scaffolds due to the distinct mineralization extent on various scaffolds. A layer of apatite coating was clearly formed on the surface of PLAGA/n-HA scaffolds. It should be noted that the apatite emerged as an aggregation of nano particles on the surface scaffolds, which makes them distinguishable from the original well-distributed nano HA particles incorporated in the scaffolds (Figures $2 \mathrm{~A}-\mathrm{a} / \mathrm{c}$ ). The size of nano HA particles were within the range of $100-300 \mathrm{~nm}$ as shown by SEM. In contrast, no apatite coating was formed on plain PLAGA scaffold and the scaffold surface appeared to be smooth after immersion in SBF for 28 days (Figure $2 \mathrm{~b} / \mathrm{d}$ ). Only at high magnification, few apatite nanoparticles were observed, indicating plain PLAGA surface did not induce mineralization in vitro.

To further prove that the apatite is newly formed through the precipitation from surrounding $\mathrm{SBF}$, composite and pure PLAGA scaffolds were stained with ALZ staining. Both PLAGA/n-HA scaffolds and PLAGA plain scaffolds were incubated in DDI water for same time period to serve as control groups. Alizarin red staining showed that PLAGA/n-HA scaffolds in DDI water exhibited no obvious staining, indicating that the incorporated n-HA were mainly embedded into the polymer matrix instead of exposing to the surface (Figure $3 \mathrm{~A}$ ). However, PLAGA/n-HA scaffolds exhibited clearly visible red staining as shown in Figure 3B after incubation in SBF for 28days, suggesting the deposition of mineral on the surface. As for plain PLAGA scaffold, no visible staining was observed in both DDI water and SBF groups, suggesting this scaffold could not initiate mineralization on its surface in vitro. We next quantified the calcium deposition by dissolving the staining in $10 \%$ CPC solution. There is no significant difference between two types of scaffolds in the initial incubation in SBF up to 14 days. From day 21, the calcium deposition on PLAGA/n-HA scaffolds increased remarkably and showed significant difference from PLAGA scaffolds. The relative amount of calcium deposition on PLAGA/n-HA was over 5-fold of the amount detected on PLAGA scaffolds (Figure $3 B$ ). These data indicate that incorporation of n-HA into the scaffolds can accelerate the mineralization of composite scaffold, in turn, improve the bioactivity in vitro.

The capability of forming apatite mineral on materials surface has been regarded as an important criterion in evaluating the bioactivity of biomaterials, especially for bone substitute materials [23]. The accelerated mineral deposition observed on PLAGA/n-HA scaffolds implied that incorporation of hydroxyapatite into the microsphere sintered scaffolds could improve their bioactivity in term of mineral deposition. The newly formed apatite layer showed characteristic morphology of tightly packed aggregation of nano particles, which has been observed by other researchers [24-26]. This unique morphology combined with Alizarin red staining confirmed that the observed mineral layer was formed during SBF incubation instead of the n-HA incorporated during scaffold fabrication. Although n-HA did not appear on composite scaffold surface, it could still promote surface mineralization by providing a local calcium rich microenvironment. It has been reported that hydroxyapatite undergoes dynamic dissolution/ re-precipitation process, thus the release of calcium ions during hydroxyapatite dissolution might create a high calcium concentration zone at the interface between scaffold and SBF [27]. This local environment might favor of apatite nucleation on the scaffold surface which then promoted the mineralization on the scaffolds. The mineral forming ability of scaffold is of particular importance as this ability predicts the performance of the scaffolds in vivo, which is to form direct bonding with natural bone. Thus, the incorporation of n-HA might improve the integration of the composite scaffolds towards host bone in vivo.

\section{Cellular response of PLAGA/n-HA composite scaffolds in vitro}

RMSCs were seeded on both types of scaffolds to evaluate the cytocompatibility of the scaffolds with and without n-HA. Cell proliferation measured by MTS assay showed that the cell numbers on both scaffolds increased with the culture time, indicating both scaffolds facilitate cell adhesion and growth. Importantly, the impact of n-HA incorporation into the scaffolds was clearly shown in cell proliferation. RMSCs seeded on PLAGA/n-HA scaffold started to proliferate earlier than PLAGA scaffold thus the proliferation of these cells plateaued earlier. In contrast, RMSCs on PLAGA grew slowly in the first week but the cell number continued to increase till day 14. The cell number on PLAGA/n-HA was substantially higher than PLAGA control

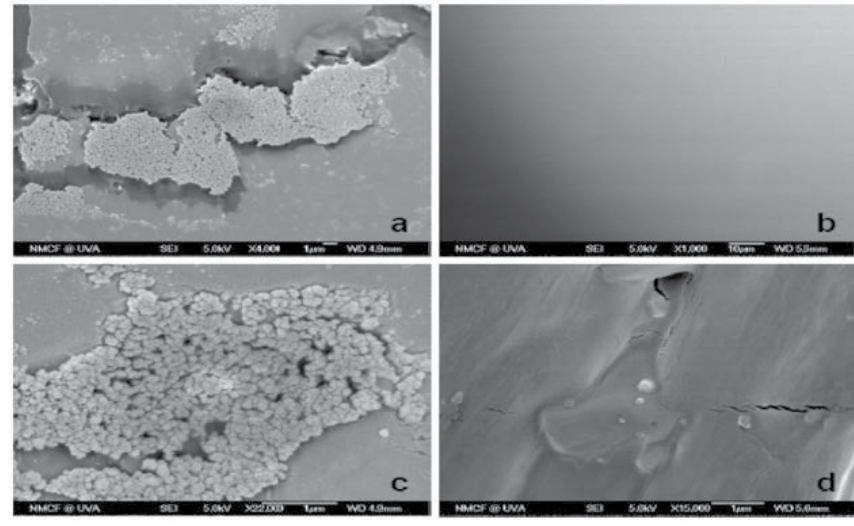

Figure 2: Morphology of scaffolds after 28 days incubation in SBF. (a) PLAGA/nHA composite scaffolds, low mag; (b)PLAGA scaffolds, low mag; (c) PLAGA/nHA, high mag; (d) )PLAGA, high mag

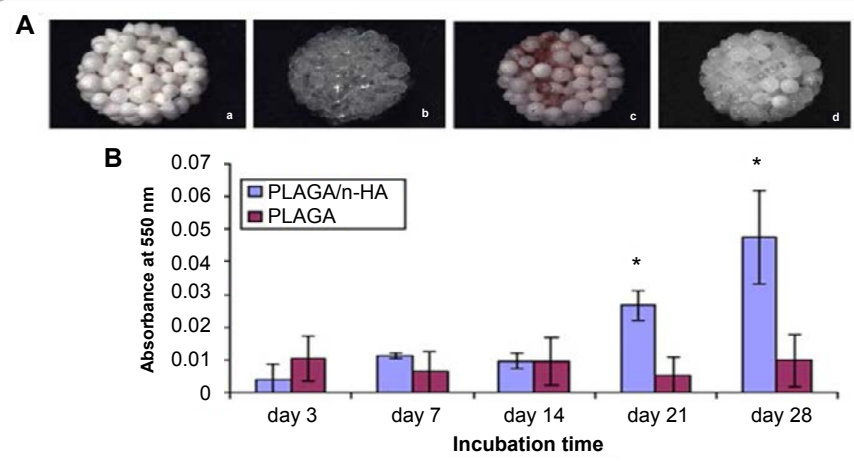

Figure 3: Evaluation of PLAGA/n-HA scaffolds mineralizationin vitro: A) Alizarin red staining of scaffolds after 28 days incubation in SBF or DDI water: (a) PLAGA/n-HA in DDI water; (b)PLAGA scaffolds in DDI water; (c) PLAGA/n-HA in SBF; (d) )PLAGA scaffolds in SBF. B) Quantification of calcium deposition from surrounding SBF as a function of incubation time. $\left(^{*}\right)$ denotes a significantly higher calcium deposition than PLAGA scaffolds at the same time point. $p=0.05$ 

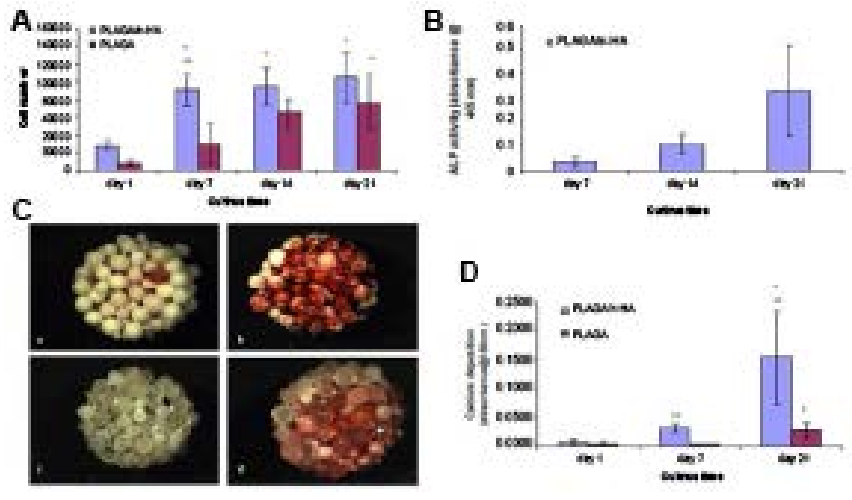

Figure 4: In vitro cytocompatibility evaluation of PLAGA/n-HA composite scaffolds:

A) RMSC proliferation measured by MTS assay;an increase in cell number was observed with increasing culture time. On day 1 and 7 , cell number on PLAGA/nHA was significantly higher than on PLAGA. $\left(^{*}\right)$ indicates significantly higher cell number than day $\left.1 .{ }^{* *}\right)$ indicates significantly higher cell number on PLAGA/n$\mathrm{HA}$ than on PLAGA at the same time point

B) ALP activity of RMSCs on PLAGA/n-HA scaffolds cultured in static condition. An increasing trend of ALP with culture time was observed.

C) Alizarin red staining of scaffolds after 21 day culture in static condition. (a) PLAGA/n-HA with no cells; (b) PLAGA/n-HA with cells; (c) blank PLAGA; (d) PLAGA scaffolds with RMSCs. RMSCs on PLAGA/n-HA and PLAGA scaffolds exhibited abundant red color after 21 day culture in static condition. D) Calcium deposition by RMSCs cultured in static condition. Calcium deposition increased with culture time on both PLAGA/n-HA and PLAGA scaffolds. On day 21, calcium deposition on PLAGA/n-HA scaffolds was significantly higher than on PLAGA scaffolds. $\left(^{*}\right)$ denotes a significantly higher calcium deposition than previous time points for the same type of scaffold. $\left({ }^{* *}\right)$ denotes a significantly higher calcium deposition than PLAGA scaffolds at the same time point.

group on day 1 and 7, indicating addition of n-HA into the scaffold might promote early stage cell adhesion and proliferation under static culture condition. However, no significant difference on cell number was found between the two tested groups on day 14 and 21 (Figure 4A). Unlike the trend of cell number on PLAGA/n-HA scaffolds, ALP activity on this scaffold exhibited an increasing tendency during the whole three-week culture period (Figure 4B). Combined this data with the previous cell proliferation results (Figure 4A), we conclude that this composite scaffold not only supported cell growth after seeding cells into the scaffold, but also promoted early stage differentiation of RMSCs towards osteogenic lineage as ALP is an early marker for osteogenic differentiation.

As one of the most important functions of osteoblastic lineage cells, deposition of calcium onto ECM is worthy of investigation as an indication of mineralization. Alizarin red staining was used to visualize the calcium deposited onto the scaffold, as shown in Figure 4C. The plain scaffolds without cells (left column) exhibited nearly no color, indicating that almost no calcium was deposited on the scaffold. After 21 days culture in static condition, RMSCs on both PLAGA/n-HA and PLAGA scaffolds demonstrated visible red staining on the surface suggesting calcium deposition took place on both scaffolds, but the staining on PLAGA/n-HA was more intense than PLAGA scaffold. Plus, we also quantified the calcium deposition by dissolving the dye in $10 \% \mathrm{CPC}$ solution and measured the OD at $550 \mathrm{~nm}$. As shown in Figure $4 \mathrm{D}$, calcium deposition gradually increased on both types of scaffolds, but the amount of calcium deposited in term of dye staining quantification was much higher in PLAGA/n-HA at day 7 and day 21. This observation suggests incorporation of n-HA could serve as a simulative cue to improve the mineralization of RMSC grown on PLAGA/n-HA.
The improvement of RMSC performance on PLAGA/n-HA could mainly be attributed to the incorporation of n-HA into the composite scaffolds. Biodegradable polymers used for tissue engineering scaffolds fabrication such as PLAGA are generally considered as non-osteoconductive, thus introduction of hydroxyapatite into polymer scaffold has been a common approach to improve the osteoconductivity of the scaffolds $[2,28]$. Here we observed that both RMSCs proliferation and differentiation was promoted after addition of n-HA into the building block of our unique microsphere based scaffolds. In particular, RMSCs proliferation on PLAGA/nHA scaffolds was accelerated in the first week of cell culture. Since it has been well documented that hydroxyapatite had extremely high affinity to a diverse range of proteins and other biomolecules, the cell proliferation of RMSC might be stimulated by the enrichment of proteins and growth factors by $\mathrm{n}-\mathrm{HA}$ on scaffold surface $[29,30]$. We also found that the osteogenic differentiation of RMSCs on the composite scaffolds was largely enhanced as shown in Figure 3B and C. n-HA trapped in PLAGA microspheres might release certain amount of calcium ions before they reach equilibrium with the local microenvironment, this might play an important role during RMSC differentiation. A recent report by Boer et al. found that free calcium ions in culture medium could trigger a calcium-inducing signaling pathway and lead to osteogenic differentiation of MSCs [31]. Thus, we speculate that the release of calcium ions from n-HA might be the driving force for the enhancement of RMSC differentiation towards osteoblasts. Therefore, our results suggest that PLAGA/n-HA substrate enhanced MSCs proliferation at early time points, while differentiation and mineralization were promoted at later time points.

\section{Conclusion}

We have successfully evaluated the bioactivity of polymeric sintered microsphere scaffolds incorporated with n-HA. Our results suggest that $\mathrm{n}$-HA was incorporated into the PLAGA microspheres with high efficiency while still maintaining the integrity of the composite scaffolds after sintering. The capability of inducing apatite formation in vitro was largely enhanced in the composite scaffolds compared to plain PLAGA scaffolds. More importantly, PLAGA/n-HA composite scaffolds have been shown to improve rabbit MSCs proliferation, differentiation, and mineralization as compared to control plain PLAGA scaffolds. Therefore, utilization of $\mathrm{n}-\mathrm{HA}$ as an additive to be introduced into polymeric microspheres could be an efficient approach to improve the bioactivity of scaffolds designed for bone tissue engineering.

\section{Acknowledgement}

This work is financially supported by National Science Foundation Grant BES 0503207 and EFRI 0736002, and National Institute of Health Grant AR063698.

\section{References}

1. Karageorgiou V, Kaplan D (2005) Porosity of 3D biomaterial scaffolds and osteogenesis. Biomaterials 26: 5474-5491.

2. Rezwan K, Chen QZ, Blaker JJ, Boccaccini AR (2006) Biodegradable and bioactive porous polymer/inorganic composite scaffolds for bone tissue engineering. Biomaterials 27: 3413-3431.

3. YuX (2012) Controlling the structural organization of regenerated bone by tailoring tissue engineering scaffold architecture. J Mater Chem 22: 9721-9730.

4. Murphy WL, Dennis RG, Kileny JL, Mooney DJ (2002) Salt fusion: An approach to improve pore interconnectivity within tissue engineering scaffolds. Tissue Eng 8: 43-52

5. Wei GB, Ma PX (2006) Macroporous and nanofibrous polymer scaffolds and polymer/bone-like apatite composite scaffolds generated by sugar spheres. $J$ Biomed Mater Res Part A 78A: 306-315. 
Citation: Lv Q, Yu X, Deng M, Nair L, Laurencin CT (2014) Evaluation of PLAGA/n-HA Composite Scaffold Bioactivity in vitro. Bioceram Dev Appl 4: 080. doi: $10.4172 / 2090-5025.1000080$

6. Lu H, Ko Y-G, Kawazoe N, Chen G (2010) Cartilage tissue engineering using funnel-like collagen sponges prepared with embossing ice particulate templates. Biomaterials 31: 5825-5835

7. Devin JE, Attawia MA, Laurencin CT (1996) Three-dimensional degradable porous polymer-ceramic matrices for use in bone repair. J Biomater Sci Polym Ed 7: 661-669.

8. Borden M, Attawia M, Khan Y, Laurencin CT (2002) Tissue engineered microsphere-based matrices for bone repair: design and evaluation. Biomaterials 23: 551-559.

9. Borden M, El-Amin SF, Attawia M, Laurencin CT (2003) Structural and human cellular assessment of a novel microsphere-based tissue engineered scaffold for bone repair. Biomaterials 24: 597-609

10. Nair LS, Laurencin CT (2007) Biodegradable polymers as biomaterials. Prog Polym Sci 32: 762-798.

11. Ulery BD, Nair LS, Laurencin CT (2011) Biomedical Applications of Biodegradable Polymers. J Polym Sci B Polym Phys 49: 832-864.

12. Jiang T, Abdel-Fattah WI, Laurencin CT (2006) In vitro evaluation of chitosan/ poly(lactic acid-glycolic acid) sintered microsphere scaffolds for bone tissue engineering. Biomaterials 27: 4894-903

13. Lu HH, Kofron MD, El-Amin SF, Attawia MA, Laurencin CT (2003) In vitro bone formation using muscle-derived cells: a new paradigm for bone tissue engineering using polymer-bone morphogenetic protein matrices. Biochem Biophys Res Commun 305: 882-9.

14. LeGeros RZ (2008) Calcium Phosphate-Based Osteoinductive Materials. Chem Rev 108: 4742-4753.

15. Wright-Charlesworth DD, King JA, Miller DM, Lim CH (2006) In vitro flexura properties of hydroxyapatite and self-reinforced poly(L-lactic acid). J Biomed Mater Res Part A 78A: 541-549.

16. Nie $\mathrm{H}$, Wang $\mathrm{CH}$ (2007) Fabrication and characterization of PLGA/HAp composite scaffolds for delivery of BMP-2 plasmid DNA. J Control Release 120: 111-121.

17. Curtin CM (2012) Innovative Collagen Nano-Hydroxyapatite Scaffolds Offer a Highly Efficient Non-Viral Gene Delivery Platform for Stem Cell-Mediated Bone Formation. Adv Mater 24: 749-754.

18. Peng $F$, Yu XH, Wei M (2011) In vitro cell performance on hydroxyapatite particles/poly(L-lactic acid) nanofibrous scaffolds with an excellent particle along nanofiber orientation. Acta Biomater 7: 2585-2592.
19. Lv Q, Deng M, Ulery BD, Nair LS, Laurencin CT (2013) Nano-ceramic composite scaffolds for bioreactor-based bone engineering. Clin Orthop Relat Res 471: 2422-33.

20. Lv Q, Nair L, Laurencin CT (2009) Fabrication, characterization, and in vitro evaluation of poly(lactic acid glycolic acid)/nano-hydroxyapatite composite microsphere-based scaffolds for bone tissue engineering in rotating bioreactors. J Biomed Mater Res A 91: 679-91

21. Yu X, Botchwey EA, Levine EM, Pollack SR, Laurencin CT (2004) Bioreactorbased bone tissue engineering: the influence of dynamic flow on osteoblast phenotypic expression and matrix mineralization. Proc Natl Acad Sci U S A 101: 11203-8.

22. Kokubo T, Takadama $\mathrm{H}$ (2006) How useful is SBF in predicting in vivo bone bioactivity? Biomaterials 27: 2907-2915.

23. Yu X, Wei M, Walsh J (2014) Covalent Immobilization of Collagen on Titanium through Polydopamine Coating to Improve Cellular Performances of MC3T3-E1 Cells. RSC Adv 4: 7185-7192.

24. Choi S, Murphy WL (2012) The effect of mineral coating morphology on mesenchymal stem cell attachment and expansion. J Mater Chem 22: 25288.

25. Yu X, Wei M (2013) Cellular Performance Comparison of Biomimetic Calcium Phosphate Coating and Alkaline-Treated Titanium Surface. Biomed Res Int 2013: $1-9$

26. Xia Z, Yu X, Wei M (2012) Biomimetic collagen/apatite coating formation on Ti6Al4V substrates. J Biomed Mater Res Part B Appl Biomater 100B: 871-881.

27. Suárez-González D (2012) Controllable mineral coatings on PCL scaffolds as carriers for growth factor release. Biomaterials 33: 713-721.

28. Hutmacher DW (2000) Scaffolds in tissue engineering bone and cartilage. Biomaterials 21: 529-2543.

29. Yu X (2014) Modulation of host osseointegration during bone regeneration by controlling exogenous stem cell differentiation using a material approach Biomater Sci 2: 242-251

30. Liu Y, Li JP, Hunziker EB, De Groot K (2006) Incorporation of growth factors into medical devices via biomimetic coatings. Philos Trans R Soc a-Mathematical Phys Eng Sci 364: 233-248.

31. Barradas AMC (2012) A calcium-induced signaling cascade leading to osteogenic differentiation of human bone marrow-derived mesenchymal stromal cells. Biomaterials 33: 3205-3215. 